\title{
Rule Calculus: Semantics, Axioms and Applications
}

\author{
Yi Zhou, Yan Zhang \\ Intelligent Systems Lab \\ School of Computing and Mathematics \\ University of Western Sydney \\ Locked Bag 1797, NSW, Australia
}

\begin{abstract}
We consider the problem of how a default rule can be deduced from a default theory. For this purpose, we propose an axiom system which precisely captures the deductive reasoning about default rules. We show that our axiomatic system is sound and complete under the semantics of the logic of here-and-there. We also study other important properties such as substitution and monotonicity of our system and prove the essential decision problem complexity. Finally, we discuss applications of our default rule calculus to various problems.
\end{abstract}

\section{Introduction}

Default logic is one of the predominant approaches for nonmonotonic reasoning. Many research topics related to default logic have been considerably studied including extensions, variations and alternatives [1-3] of Reiter's original definition [4], computational issues $[5,6]$ and so on.

However, one problem in default logic has been neglected in previous research. That is, how can we deduce a default rule from a default theory? In other words, in which sense can we say that a default rule is a consequence of a given default theory? This problem of rule deduction is of special interests from both theoretical and practical viewpoints. For instance, we may consider whether we can have a deductive system to formalize reasoning about default rules, and also implement a nonmonotonic knowledge system for more complex decision making where a decision could be a default rule. Quite obviously, to achieve such goals, the first fundamental task is that we should develop a logic or calculus for default rule reasoning.

In this paper, we propose a logical calculus, called default rule calculus (rule calculus for short), to address the problem of rule deduction. We first extend the logic of here-and-there to define a model-theoretical semantics for rule calculus, and discuss its relationships to the extension semantics. Then we define an axiom system, which extends both classical propositional calculus and the intermediate logic G3 (Gödel's 3 -valued logic) [7], and prove its soundness and completeness. We further investigate some important properties of our system such as substitution and monotonicity, and prove the essential decision problem complexity. Finally, we discuss how our work can be applied to various problems such as the extension of generality among default rules and revision of nonmonotonic knowledge bases.

The reasons why we use the logic of here-and-there are as follows. Firstly, it is well studied in philosophical logic and it also has a simple axiomatic counterpart, namely G3 
[7]. Secondly, it is proven to be a very useful foundation of answer set programming [2, 8-10]. As pointed out in [3], answer set programming is a special case of default logic by restricting the propositional formulas to atoms. Thus, the extended version of the logic of here-and-there should, analogously, serve as a foundation of default logic. Thirdly, the logic of here-and-there naturally captures $[2,11]$ the notion of strong equivalence [11], which is argued to be the notion of "real equivalence" among answer set programs. As a consequence, it will also capture real equivalence among default rules.

In this paper, we will use general default logic [3] as a basis for the development of default rule calculus. Reasons for this are of three aspects: firstly, general default logic is a generalization of Reiter's default logic [4], Gelfond et al.'s disjunctive default logic [1] and Turner's nested default logic [2], which provides the most generalized default reasoning in the default logics paradigm; secondly, the syntax of general default logic is defined as arbitrary compositions of propositional formulas and rule connectives; finally, its extension semantics is defined in a very simple way as that for answer set programming [9].

The rest of the paper is organized as follows. In Section 2, we briefly review the syntax and semantics of general default logic, and then define the semantics of rule calculus. In Section 3, we present an axiom system for rule calculus and prove its soundness and completeness result. We then study relevant important properties of our axiom system for default rule calculus in Section 4. In Section 5, we discuss possible applications of rule calculus. Finally, in Section 6 we conclude the paper with some remarks.

\section{Rule calculus: syntax and semantics}

To begin with, we recall some basic notions of classical propositional logic. The classical propositional language $\mathcal{L}$ is defined recursively by a set Atom of atoms (or primitive propositions, variables) and a set of classical connectives $\perp, \rightarrow$ and $\neg$. Other connectives, such as $T, \wedge, \vee, \leftrightarrow$, are defined as usual. Literals are atoms and their negations. The satisfaction relation $\models$ is defined as usual. A set of formulas in $\mathcal{L}$ is said to be a theory iff it is closed under classical entailment. Moreover, it is inconsistent iff it contains both a formula $F$ and $\neg F$, otherwise, it is consistent. Let $\Gamma$ be a set of formulas, by $T h(\Gamma)$ we denote the theory containing all formulas entailed by $\Gamma$. For convenience, we also use a set of formulas $\Gamma$ to denote a theory $T$ if $T=T h(\Gamma)$.

The language $\mathcal{R}$ of general default logic [3] is defined upon $\mathcal{L}$ by adding a set of rule connectives $\Rightarrow, \&$ and $\mid$ recursively:

$$
R::=F|R \Rightarrow R| R \& R|R| R,
$$

where $F \in \mathcal{L}$. $-R$ and $R_{1} \Leftrightarrow R_{2}$ are considered as shorthand of $R \Rightarrow \perp$ and $\left(R_{1} \Rightarrow\right.$ $\left.R_{2}\right) \&\left(R_{2} \Rightarrow R_{1}\right)$ respectively. The order of priority for these connectives are

$$
\{\neg\}>\{\wedge, \vee\}>\{\rightarrow, \leftrightarrow\}>\{-\}>\{\&, \mid\}>\{\Rightarrow, \Leftrightarrow\} .
$$

Formulas in $\mathcal{R}$ are called rules, whilst formulas in $\mathcal{L}$ are called facts. A rule base is a set of rules. The satisfaction relation $\models$ between a theory $T$ and a rule $R$ is defined recursively as follows: 
- If $R$ is a fact, then $T \models R$ iff $R \in T$.

$-T \models R \& S$ iff $T \models R$ and $T \models S$;

$-T \models R \mid S$ iff $T \models R$ or $T \models S$;

$-T \models R \Rightarrow S$ iff $T \not R$ or $T \models S$.

Hence, if $T$ is consistent, then $T \models-R$ iff $T \not \models R$. If $T$ is inconsistent, then for every rule $R, T \models R$. We say that $T$ is a model of $R$ iff $T \models R$.

The extension semantics of general default logic defined in [3] is not defined as the same as Reiter's original definition [4]. However, it is defined in a reduction-style similarly to that of answer set programming [9]. The reduct of a rule $R$ relative to a theory $T$, denoted by $R^{T}$, is the rule obtained from $R$ by replacing every maximal subrule $^{1}$ of $R$ which is not satisfied by $T$ with $\perp$. The reduct of a rule base relative to a theory is defined as the set of reducts of its rules relative to this theory. A theory $T$ is said to be an extension of a rule base $\Delta$ iff it is the minimal (in the sense of set inclusion) theory satisfying $\Delta^{T}$.

As shown in [3], Reiter's default logic [4] in propositional case is a special case of general default logic by restricting the rules to the following form

$$
F \&-G_{1} \& \ldots \&-G_{n} \Rightarrow H
$$

where $n \geq 0, F, G_{i},(1 \leq i \leq n)$ and $H$ are facts. Yet, under the context of Reiter's default logic, this form is represented as

$$
\frac{F: M\left(\neg G_{1}\right), \ldots, M\left(\neg G_{n}\right)}{H} .
$$

Similarly, both Gelfond et al.'s disjunctive default logic [1] and Turner's nested default logic [2] are also special cases of general default logic.

Here, we adopt Turner's (Section 7 in [2]) extended notion of Heyting's logic of here-and-there, introduced by Pearce [10] into answer set programming, as the basic semantics for general default logic. An HT-interpretation is a pair $\left\langle T_{1}, T_{2}\right\rangle$, where $T_{1}$ and $T_{2}$ are theories such that $T_{1} \subseteq T_{2}$. The satisfaction relation $\models^{2}$ between an HTinterpretation $\left\langle T_{1}, T_{2}\right\rangle$ and a rule $R$ is defined recursively:

- for a fact $F,\left\langle T_{1}, T_{2}\right\rangle \models F$ iff $F \in T_{1}$;

$-\left\langle T_{1}, T_{2}\right\rangle \models R_{1} \& R_{2}$ iff $\left\langle T_{1}, T_{2}\right\rangle \models R_{1}$ and $\left\langle T_{1}, T_{2}\right\rangle \models R_{2}$;

$-\left\langle T_{1}, T_{2}\right\rangle \models R_{1} \mid R_{2}$ iff $\left\langle T_{1}, T_{2}\right\rangle \models R_{1}$ or $\left\langle T_{1}, T_{2}\right\rangle \models R_{2}$;

$-\left\langle T_{1}, T_{2}\right\rangle \models R_{1} \Rightarrow R_{2}$ iff

1. $\left\langle T_{1}, T_{2}\right\rangle \not \models R_{1}$ or $\left\langle T_{1}, T_{2}\right\rangle \models R_{2}$, and

2. $T_{2} \models R_{1} \Rightarrow R_{2}$.

We say that $\left\langle T_{1}, T_{2}\right\rangle$ is an HT-model of a rule $R$ iff $\left\langle T_{1}, T_{2}\right\rangle \models R$. We say that a rule base $\Delta$ implies a rule $R$, denoted by $\Delta \models R$, iff all HT-models of $\Delta$ are also HT-models of $R$. It is easy to see that the HT-interpretation $\langle\perp, \perp\rangle$ is a model of all rules. We say

\footnotetext{
${ }^{1}$ The subrule relation is defined recursively: a) $R_{1}$ is a subrule of $R_{1}$, and b) $R_{1}$ and $R_{2}$ are subrules of $R_{1} \& R_{2}, R_{1} \mid R_{2}$ and $R_{1} \Rightarrow R_{2}$.

${ }^{2}$ For convenience, we overload the notation $\models$ in this paper.
} 
that a rule $R$ is a rule contradiction iff $\langle\perp, \perp\rangle$ is the only HT-model of $R$. We say that a rule $R$ is a rule tautology iff every HT-interpretation is an HT-model of $R$.

Intuitively, a theory $T$ is a possible set of information of an agent about the world, and a rule $R$ is a description or constraint about the information of the world. $T \models R$ means that the set $T$ of information obeys (or does not violate) the constraint $R ; T$ is an extension of $R$ means that $T$ is one of the possible sets of information can be derived by giving the only constraint $R$. Given a rule base $\Delta$ and a rule $R, \Delta \models R$ means that the set of constraints $\Delta$ is more powerful than the constraint $R$. In other words, $R$ can be eliminated by giving $\Delta$.

Example 1. Consider the notorious bird-fly example. The statement "birds normally fly" can be represented as a default rule bird\& $-\neg f l y \Rightarrow f l y$. Given an instance of bird, represented as a fact bird, by the extension semantics, the only extension of the rule base $\{$ bird \& $-\neg f l y \Rightarrow f l y$, bird $\}$ is $\{$ bird, fly $\}$. However, fly is not a rule consequence of the rule base $\{$ bird \& $-\neg f l y \Rightarrow f l y$, bird $\}$ since $\langle\{$ bird $\},\{$ bird, $\neg f l y\}\rangle$ is an HTmodel of $\{$ bird \& $-\neg f l y \Rightarrow f l y$, bird $\}$ but not an HT model of $\{$ bird, fly $\}$. This shows a difference between the extension semantics and the HT-semantics.

Similarly, bird \& $-\neg f l y \Rightarrow f l y \not=$ bird $\Rightarrow f l y$. However, one can check that all HT-models of bird $\Rightarrow f l y$ are also HT-models of bird \& $-\neg f l y \Rightarrow f l y$. Therefore, bird $\Rightarrow f l y \models$ bird \& $-\neg f l y \Rightarrow f l y$. This means that, intuitively, the statement "birds normal fly" is strictly weaker than the statement "birds fly".

Example 2. Let $p_{1}, p_{2}$ and $p_{3}$ be three atoms. Consider the rule base $\left\{p_{1} \&-\neg p_{2} \Rightarrow\right.$ $\left.p_{2}, p_{2} \&-\neg p_{3} \Rightarrow p_{3}\right\}$, which has an HT-model $\left\langle\left\{p_{1}\right\},\left\{p_{1}, \neg p_{2}\right\}\right\rangle$. However, this HTinterpretation is not an HT-model of $p_{1} \&-\neg p_{3} \Rightarrow p_{3}$. This shows that $\left\{p_{1} \&-\neg p_{2} \Rightarrow\right.$ $\left.p_{2}, p_{2} \&-\neg p_{3} \Rightarrow p_{3}\right\} \not \models p_{1} \&-\neg p_{3} \Rightarrow p_{3}$.

The extension semantics and HT-semantics of general default logic are closely related.

Proposition 1. Let $T_{1}$ and $T_{2}$ be two consistent theories such that $T_{1} \subseteq T_{2}$ and $R$ a rule.

$-T_{1} \models R$ iff $\left\langle T_{1}, T_{1}\right\rangle \models R$.

- If $\left\langle T_{1}, T_{2}\right\rangle \models R$, then $T_{2} \models R$.

$-\left\langle T_{1}, T_{2}\right\rangle \models-R$ iff $T_{2} \models-R$.

Proposition 2. Let $\Delta$ be a rule base and $F$ a fact. If $\Delta \models F$, then $F$ is in all extensions of $\Delta$.

However, the converse of Proposition 2 does not hold in general. For instance, $\left\{p_{1}\right\}$ is the unique extension of $-p_{2} \Rightarrow p_{1}$. Thus, $p_{1}$ is in all extensions of $-p_{2} \Rightarrow p_{1}$. However, $-p_{2} \Rightarrow p_{1} \not \models p_{1}$ since $\left\langle\left\{p_{2}\right\},\left\{p_{2}\right\}\right\rangle$ is an HT-model of $-p_{2} \Rightarrow p_{1}$ but not an HT-model of $p_{1}$.

Proposition 3. Let $T_{1}$ and $T_{2}$ be two theories such that $T_{1} \subseteq T_{2}$ and $\Delta$ a rule base. $\left\langle T_{1}, T_{2}\right\rangle$ is an HT-model of $\Delta$ iff $T_{1} \models \Delta^{T_{2}}$. 
Proposition 4. Let $T$ be a theory and $\Delta$ a rule base. $T$ is an extension of $\Delta$ iff $\langle T, T\rangle$ is an HT-model of $\Delta$, and for all theories $T_{1} \subset T,\left\langle T_{1}, T\right\rangle$ is not an HT-model of $\Delta$.

The notion of strong equivalence, introduced by [11] into answer set programming, plays an important role from both a theoretical and a practical viewpoints. A similar notion is introduced into default logic in [2]. We say that two rules $R_{1}$ and $R_{2}$ are strongly equivalent, denoted by $R_{1} \equiv R_{2}$, iff for all other rules $R_{3}, R_{1} \& R_{3}$ has the same set of extensions as $R_{2} \& R_{3}$. Strong equivalence can also be defined in another way. That is, two rules $R_{1}$ and $R_{2}$ are strongly equivalent iff for all other rules $R_{3}, R_{3}$ has the same set of extensions as $R_{3}\left(R_{1} / R_{2}\right)$, where $R_{3}\left(R_{1} / R_{2}\right)$ is the rule obtained from $R_{3}$ by replacing every occurrence of $R_{1}$ in $R_{3}$ with $R_{2}$ simultaneously. It is clear that the notion of strong equivalence can be extended for the cases of rule bases.

In fact, strong equivalence in general default logic can be captured in the logic of here-and-there.

Proposition 5. Let $R_{1}$ and $R_{2}$ be two rules. $R_{1}$ and $R_{2}$ are strongly equivalent iff they have the same set of HT-models in the logic of here-and-there. That is, $R_{1} \equiv R_{2}$ iff $\models R_{1} \Leftrightarrow R_{2}$.

Since general default logic is both an extension of general logic programming and nested default logic, Proposition 5 is a generalization of both Proposition 2 in [9] and Theorem 3 in [2]. As a consequence of Proposition 5, checking whether a rule is implied by a rule base can be reduced to checking whether two rule bases are strongly equivalent.

Corollary 1. Let $\Delta$ be a rule base and $R$ a rule. $\Delta \models R$ iff $\Delta \cup\{R\}$ is strongly equivalent to $\Delta$.

Corollary 1 indicates the intuition behind rule deduction, that is, a rule $R$ is a consequence of a rule base $\Delta$ means that $R$ provides no more information by giving $\Delta$. In other words, $R$ can be eliminated by giving $\Delta$.

\section{Rule calculus: axiom system}

In this section, we propose an axiom system for default rule calculus and prove the soundness and completeness results.

Axioms The axioms of rule calculus are:

A1 all tautologies in classical propositional logic.

A2 $\left(F_{1} \rightarrow F_{2}\right) \Rightarrow\left(F_{1} \Rightarrow F_{2}\right)$, where $F_{1}$ and $F_{2}$ are two facts.

A3 $R_{1} \Rightarrow\left(R_{2} \Rightarrow R_{1}\right)$.

A4 $\left(R_{1} \Rightarrow\left(R_{2} \Rightarrow R_{3}\right)\right) \Rightarrow\left(\left(R_{1} \Rightarrow R_{2}\right) \Rightarrow\left(R_{1} \Rightarrow R_{3}\right)\right)$.

A5 $R_{1} \Rightarrow\left(R_{2} \Rightarrow\left(R_{1} \& R_{2}\right)\right)$.

A6 $R_{1} \& R_{2} \Rightarrow R_{1} ; R_{1} \& R_{2} \Rightarrow R_{2}$.

A7 $R_{1} \Rightarrow R_{1}\left|R_{2} ; R_{2} \Rightarrow R_{1}\right| R_{2}$.

A8 $\left(R_{1} \Rightarrow R_{3}\right) \Rightarrow\left(\left(R_{2} \Rightarrow R_{3}\right) \Rightarrow\left(R_{1} \mid R_{2} \Rightarrow R_{3}\right)\right)$.

A9 $\left(R_{1} \Rightarrow R_{2}\right) \Rightarrow\left(\left(R_{1} \Rightarrow-R_{2}\right) \Rightarrow-R_{1}\right)$.

A10 $R_{1}\left|\left(R_{1} \Rightarrow R_{2}\right)\right|-R_{2}$. 
Rules The only ${ }^{3}$ inference rule of rule calculus is

Rule Modus Ponens from $R_{1}$ and $R_{1} \Rightarrow R_{2}$ to infer $R_{2}$.

Axiom 1 simply means that all classical tautologies are also rule tautologies; Axiom 2 is for bridging the gap between facts and rules; Axiom 3-9, together with Rule Modus Ponens, are generalization of the axiom system of intuitionistic logic [12]; Axiom A10 is the extended version of an additional axiom in the intermediate logic G3. That is, Axiom 3-10 and Rule Modus Ponens are generalization of the axiom system of G3 [10].

A rule $R$ is said to be a consequence of a rule base $\Delta$, denoted by $\Delta \vdash R$, iff there is a sequence of rules $R_{1}, \ldots, R_{n}$ such that $R_{n}=R$ and for each $i,(1 \leq i \leq n)$, either a) $R_{i}$ is an instance of axiom, or b) $R_{i}$ is in $\Delta$, or c) $R_{i}$ is obtained by an inference rule from some proceeding rules in this sequence. Such a sequence is called a proof (or deduction) of $R$ from $\Delta$. rules in $\Delta$ are called premises. A rule $R$ is said to be a rule theorem, denoted by $\vdash R$, iff there exists a proof of $R$ from the empty rule base. We use $\Delta \nvdash R$ to denote it is not the case that $\Delta \vdash R$.

As an example, we prove the following rule theorem.

Proposition 6. $\vdash\left(F_{1} \rightarrow F_{2}\right) \Rightarrow\left(F_{1} \Rightarrow-\neg F_{2}\right)$, where $F_{1}$ and $F_{2}$ are two facts.

Proof. We construct a proof as follows:

1. $\left(\neg F_{2} \rightarrow \perp\right) \Rightarrow\left(\neg F_{2} \Rightarrow \perp\right)$ by A2,

2. $\left(F_{2} \Rightarrow-\neg F_{2}\right) \Rightarrow\left(F_{1} \Rightarrow\left(F_{2} \Rightarrow-\neg F_{2}\right)\right)$ by A3,

3. $F_{1} \Rightarrow\left(F_{2} \Rightarrow-\neg F_{2}\right)$ by 1,2 and RMP,

4. $\left(F_{1} \Rightarrow\left(F_{2} \Rightarrow-\neg F_{2}\right)\right) \Rightarrow\left(\left(F_{1} \Rightarrow F_{2}\right) \Rightarrow\left(F_{1} \Rightarrow-\neg F_{2}\right)\right)$ by A4,

5. $\left(F_{1} \Rightarrow F_{2}\right) \Rightarrow\left(F_{1} \Rightarrow-\neg F_{2}\right)$ by 3,4 and RMP,

6. $\left(\left(F_{1} \Rightarrow F_{2}\right) \Rightarrow\left(\left(F_{1} \Rightarrow-\neg F_{2}\right)\right) \Rightarrow\left(\left(F_{1} \rightarrow F_{2}\right) \Rightarrow\left(\left(F_{1} \Rightarrow F_{2}\right) \Rightarrow\left(F_{1} \Rightarrow\right.\right.\right.\right.$ $\left.\left.\left.\left.-\neg F_{2}\right)\right)\right)\right)$ by A3,

7. $\left(F_{1} \rightarrow F_{2}\right) \Rightarrow\left(\left(F_{1} \Rightarrow F_{2}\right) \Rightarrow\left(F_{1} \Rightarrow-\neg F_{2}\right)\right)$ by 5, 6 and RMP,

8. $\left(\left(F_{1} \rightarrow F_{2}\right) \Rightarrow\left(\left(F_{1} \Rightarrow F_{2}\right) \Rightarrow\left(F_{1} \Rightarrow-\neg F_{2}\right)\right)\right) \Rightarrow\left(\left(\left(F_{1} \rightarrow F_{2}\right) \Rightarrow\left(F_{1} \Rightarrow\right.\right.\right.$ $\left.\left.\left.F_{2}\right)\right) \Rightarrow\left(\left(F_{1} \rightarrow F_{2}\right) \Rightarrow\left(F_{1} \Rightarrow-\neg F_{2}\right)\right)\right)$ by A4,

9. $\left(\left(F_{1} \rightarrow F_{2}\right) \Rightarrow\left(F_{1} \Rightarrow F_{2}\right)\right) \Rightarrow\left(\left(F_{1} \rightarrow F_{2}\right) \Rightarrow\left(F_{1} \Rightarrow-\neg F_{2}\right)\right)$ by 7,8 and RMP,

10. $\left(F_{1} \rightarrow F_{2}\right) \Rightarrow\left(F_{1} \Rightarrow F_{2}\right)$ by A2,

11. $\left(F_{1} \rightarrow F_{2}\right) \Rightarrow\left(F_{1} \Rightarrow-\neg F_{2}\right)$ by 9,10 and RMP.

This completes the proof.

A simple property following from the definition of proof of rule calculus is so-called compactness as follows.

Proposition 7 (Compactness). Let $\Delta$ be a rule base and $R$ a rule such that $\Delta \vdash R$. There exists a finite subset $\Delta^{\prime}$ of $\Delta$ such that $\Delta^{\prime} \vdash R$.

Proposition 8 (Deduction theorem). Let $\Delta$ be a rule base and $R_{1}$ and $R_{2}$ two rules. $\Delta \cup\left\{R_{1}\right\} \vdash R_{2}$ iff $\Delta \vdash R_{1} \Rightarrow R_{2}$.

\footnotetext{
${ }^{3}$ In fact, the Modus Ponens rule in classical logic is, of course, also an inference rule in rule calculus. However, since axiom A1 takes all classical tautologies into account, we can omit the classical Modus Ponens rule here.
} 
Deduction theorem is a very useful tool for proving the consequence relationships in rule calculus. Consider the following example.

Example 3. [Example 1 continued] We prove that bird $\Rightarrow f l y \vdash$ bird\& $-\neg f l y \Rightarrow f l y$. By deduction theorem, we only need to prove $\{$ bird $\Rightarrow f l y$, bird \& $-\neg f l y\} \vdash f l y$. This is quite simple from A6 and RMP.

Proposition 9. $\vdash\left(R_{1} \Rightarrow R_{2}\right) \Rightarrow\left(-R_{2} \Rightarrow-R_{1}\right)$.

Proof. We construct a proof of $-R_{1}$ from $\left\{R_{1} \Rightarrow R_{2},-R_{2}\right\}$.

1. $-R_{2} \Rightarrow\left(R_{1} \Rightarrow-R_{2}\right)$ by $\mathrm{A} 3$,

2. $-R_{2}$ by premises,

3. $R_{1} \Rightarrow-R_{2}$ by 1,2 and RMP,

4. $\left(R_{1} \Rightarrow R_{2}\right) \Rightarrow\left(\left(R_{1} \Rightarrow-R_{2}\right) \Rightarrow-R_{1}\right)$ by $\mathrm{A} 9$,

5. $R_{1} \Rightarrow R_{2}$ by premises,

6. $\left(R_{1} \Rightarrow-R_{2}\right) \Rightarrow-R_{1}$ by 4,5 and RMP,

7. $-R_{1}$ by 3,6 and RMP.

Thus, $\left\{R_{1} \Rightarrow R_{2},-R_{2}\right\} \vdash-R_{1}$. By deduction theorem, $\left\{R_{1} \Rightarrow R_{2}\right\} \vdash-R_{2} \Rightarrow-R_{1}$. Again, by deduction theorem, $\vdash\left(R_{1} \Rightarrow R_{2}\right) \Rightarrow\left(-R_{2} \Rightarrow-R_{1}\right)$.

Proposition 10. Let $F, G$ and $Q$ be three facts.

$$
\begin{aligned}
& \text { 1. } \vdash F \Rightarrow-\neg F . \\
& \text { 2. } \vdash(F \& G) \Leftrightarrow(F \wedge G) . \\
& \text { 3. } \vdash(-F \mid-G) \Leftrightarrow(\neg F \vee \neg G) . \\
& \text { 4. } \vdash(F \wedge Q \rightarrow G) \Rightarrow(F \&-G \Rightarrow-Q) . \\
& \text { 5. } \vdash(F \rightarrow G) \Rightarrow(-G \Rightarrow-F) .
\end{aligned}
$$

Theorem 1 (Soundness and completeness). Let $R$ be a rule. $R$ is a rule tautology iff $R$ is a rule theorem. That is, $\models R$ iff $\vdash R$.

Proof. "soundness:" We first show that all instances of axioms are rule tautologies. As an example, we only present the proofs of A2 and A10 here. Let $\left\langle T_{1}, T_{2}\right\rangle$ be an HTinterpretation other than $\langle\perp, \perp\rangle$.

A2 Assume that $\left\langle T_{1}, T_{2}\right\rangle$ is not an HT-model of $\left(F_{1} \rightarrow F_{2}\right) \Rightarrow\left(F_{1} \Rightarrow F_{2}\right)$. Then, there are two cases. Case 1: $\left\langle T_{1}, T_{2}\right\rangle \models F_{1} \rightarrow F_{2}$ and $\left\langle T_{1}, T_{2}\right\rangle \not F_{1} \Rightarrow F_{2}$. That is, $T_{1} \models F_{1} \rightarrow F_{2}$ and a) $\left\langle T_{1}, T_{2}\right\rangle \models F_{1}$ and $\left\langle T_{1}, T_{2}\right\rangle \not \models F_{2}$ or b) $T_{2} \not \models F_{1} \Rightarrow F_{2}$. Thus, $T_{1} \models F_{1} \rightarrow F_{2}$ and a) $T_{1} \models F_{1}$ and $T_{1} \not \models F_{2}$ or b) $T_{2} \models F_{1}$ and $T_{2} \not \models F_{2}$. Whichever the case is, it leads to a contradiction. Case 2: $T_{2} \forall \forall\left(F_{1} \rightarrow F_{2}\right) \Rightarrow$ $\left(F_{1} \Rightarrow F_{2}\right)$. Then, $T_{2} \models F_{1} \rightarrow F_{2}$ and $T_{2} \not \models F_{1} \Rightarrow F_{2}$. That is, $T_{2} \models F_{1} \rightarrow F_{2}$ and $T_{2} \models F_{1}$ and $T_{2} \not \models F_{2}$, a contradiction.

A10 Assume that $\left\langle T_{1}, T_{2}\right\rangle$ is not an HT-model of $R_{1}\left|\left(R_{1} \Rightarrow R_{2}\right)\right|-R_{2}$. Then, $\left\langle T_{1}, T_{2}\right\rangle \not \forall R_{1}$ and $\left\langle T_{1}, T_{2}\right\rangle \not \forall R_{1} \Rightarrow R_{2}$. Therefore $T_{2} \not \forall R_{1} \Rightarrow R_{2}$. Thus, $T_{2} \forall \forall R_{2}$. However, $\left\langle T_{1}, T_{2}\right\rangle \forall-R_{2}$. Thus, By Proposition $1, T_{2} \forall \forall-R_{2}$, a contradiction. 
We then show that all inferences rules preserve rule tautologies. Suppose that both $R_{1}$ and $R_{1} \Rightarrow R_{2}$ are rule tautologies. Given an HT-interpretation $\left\langle T_{1}, T_{2}\right\rangle$, we have that $\left\langle T_{1}, T_{2}\right\rangle \models R_{1}$ and $\left\langle T_{1}, T_{2}\right\rangle \models R_{1} \Rightarrow R_{2}$. Thus, $\left\langle T_{1}, T_{2}\right\rangle \models R_{2}$. This shows that $R_{2}$ is also a rule tautology. Hence, soundness holds.

"completeness:" As recently shown in [8], each formula in the logic of here-andthere is equivalent to a set of formulas of the following form:

$$
p_{1} \wedge \ldots \wedge p_{n} \wedge \neg p_{n+1} \wedge \ldots \wedge \neg p_{m} \rightarrow p_{m+1} \vee \ldots \vee p_{k} \vee \neg p_{k+1} \vee \ldots \vee \neg p_{l},
$$

where $p_{i},(1 \leq i \leq l)$ are atoms. A similar result for rule calculus can be proved in the same way. That is, each rule is equivalent to a set of rules of the following form:

$$
F_{1} \& \ldots \& F_{n} \&-F_{n+1} \& \ldots \&-F_{m} \Rightarrow F_{m+1}|\ldots| F_{k}\left|-F_{k+1}\right| \ldots \mid-F_{l}
$$

where $F_{i},(1 \leq i \leq l)$ are facts.

Thus, we only need to prove that for each rule $R$ of form (1), if $\models R$, then $\vdash R$. For convenience, we assume that

$$
R=F_{1} \& \ldots \& F_{n} \&-G_{1} \& \ldots \&-G_{m} \Rightarrow P_{1}|\ldots| P_{k}\left|-Q_{1}\right| \ldots \mid-Q_{l} .
$$

Let $F=\bigwedge_{1 \leq i \leq n} F_{i}$ and $Q=\bigwedge_{1 \leq i \leq l} Q_{i}$ Then, one of the following statements must hold.

1. There exists $i,(1 \leq i \leq k)$ such that $F \rightarrow P_{i}$ is a classical tautology.

2. $F \rightarrow \neg Q$ is a classical tautology.

3. There exists $j,(1 \leq j \leq m)$ such that $(F \wedge Q) \rightarrow G_{j}$ is a classical tautology.

Suppose otherwise, then there exists a propositional assignment $\pi_{0}$ such that $\pi_{0} \models F \wedge$ $Q$; there exists a propositional assignment $\pi_{i},(1 \leq i \leq k)$ such that $\pi_{i} \models F \wedge \neg P_{i}$; there exists a propositional assignment $\pi_{j}^{\prime},(1 \leq i \leq m)$ such that $\pi_{j}^{\prime} \models F \wedge Q \wedge \neg G_{j}$. Let $T_{1}$ be the theory such that the set of its models is $\left\{\pi_{0}, \pi_{i}, \pi_{j}^{\prime},(1 \leq i \leq k),(1 \leq j \leq l)\right\}$, and $T_{2}$ be the theory such that the set of its models is $\left\{\pi_{j}^{\prime},(1 \leq j \leq l)\right\}$. It is easy to check that $\left\langle T_{1}, T_{2}\right\rangle$ is not an HT-model of $R$, a contradiction.

Thus, one of the three previous statements holds. Hence, $R$ can be proved according to axioms and Proposition 6, Proposition 9 and Proposition 10. As an example, we prove the third case. Without loss of generality, suppose that $(F \wedge Q) \rightarrow G_{1}$ is a classical tautology. Then, by point 4 in Proposition $10, F \&-G_{1} \Rightarrow-Q$ is a rule tautology. Then, by point 2 and point 3 in Proposition 10, $F_{1} \& \ldots \& F_{n} \&-G_{1} \Rightarrow-Q_{1}|\ldots|-Q_{l}$ is a rule tautology. By $\mathrm{A} 6$ and $\mathrm{A} 7, R$ is a rule tautology.

From compactness, deduction theorem and soundness and completeness, we have the following result.

Corollary 2. Let $\Delta$ be a rule base and $R$ a rule. $R$ is implied by $\Delta$ iff $R$ is a consequence of $\Delta$. That is, $\Delta \models R$ iff $\Delta \vdash R$. 


\section{Other properties}

In this section, we discuss some other important properties of rule calculus.

From Proposition 10, one may claim that rule connectives and corresponding classical connectives play the same roles to some extent. However, this is not the case. For instance, $\forall(F \mid G) \Leftrightarrow(F \vee G)$, where $F$ and $G$ are two facts. As another example, from A2, $\vdash(F \rightarrow G) \Rightarrow(F \Rightarrow G)$. However, $\forall(F \Rightarrow G) \Rightarrow(F \rightarrow G)$. Consequently, $\forall-F \Rightarrow \neg F$ although $\vdash \neg F \Rightarrow-F$.

In fact, rule calculus is more like the intermediate logic G3. It is clear that the former is an extension of the latter. Hence, theorems not in G3 are not rule theorems in rule calculus. For example, $R \mid-R$ is not a rule theorem. On the other hand, theorems in $\mathrm{G} 3$ can be extended for rule calculus. For example, the following property holds.

Proposition 11. Let $R_{1}, R_{2}, R_{3}$ and $R_{4}$ be four rules.

1. $\left\{R_{1} \Rightarrow R_{2}, R_{2} \Rightarrow R_{3}\right\} \vdash R_{1} \Rightarrow R_{3}$.

2. $\left\{R_{1} \Rightarrow R_{2}, R_{3} \Rightarrow R_{4}\right\} \vdash R_{1}\left|R_{3} \Rightarrow R_{2}\right| R_{4}$.

3. $R_{1} \Rightarrow--R_{1}$.

Proposition 12. Let $R$ be a theorem in $G 3$ composed from a set of atoms $P=\left\{p_{1}, \ldots, p_{n}\right\}$ and $\Delta=\left\{R_{i},(1 \leq i \leq n)\right\}$ are $n$ rules associated with each $p_{i}$. Then, $R(P / \Delta)$ is a rule theorem, where $R(P / \Delta)$ is the rule obtained from $R$ by replacing every occurrence of $p_{i},(1 \leq i \leq n)$ with corresponding $R_{i}$ simultaneously.

Proposition 13. Let $F_{1}$ and $F_{2}$ be two facts. $F_{1} \mid F_{2} \vdash F_{1} \vee F_{2}$.

However, $F_{1} \vee F_{2} \nvdash F_{1} \mid F_{2}$. For example, $\left\langle\left\{F_{1} \vee F_{2}\right\},\left\{F_{1} \vee F_{2}\right\}\right\rangle$ is an HT-model of $F_{1} \vee F_{2}$ but not an HT-model of $F_{1} \mid F_{2}$.

Proposition 14 (Substitution). Let $R$ be a rule theorem and $R_{1}$ and $R_{2}$ two rules. $R\left(R_{1} / R_{2}\right)$, the rule obtained from $R$ by replacing every occurrence of $R_{1}$ with $R_{2}$ simultaneously, is a rule theorem as well.

Proposition 15. Let $F_{1}, F_{2}$ and $F_{3}$ be three facts. $\left\{F_{1} \Rightarrow F_{2}, F_{2} \&-\neg F_{3} \Rightarrow F_{3}\right\} \vdash$ $F_{1} \&-\neg F_{3} \Rightarrow F_{3}$.

However, $\left\{F_{2} \Rightarrow F_{3}, F_{1} \&-\neg F_{2} \Rightarrow F_{2}\right\} \not F_{1} \&-\neg F_{3} \Rightarrow F_{3}$ since $\left\langle\left\{p_{1}\right\},\left\{p_{1} \wedge \neg p_{2}\right\}\right\rangle$ is an HT-models of $\left\{p_{2} \Rightarrow p_{3}, p_{1} \&-\neg p_{2} \Rightarrow p_{2}\right\}$ but not an HT-models of $p_{1} \&-\neg p_{3} \Rightarrow$ $p_{3}$. In addition, as shown in Example 2, $\left\{F_{1} \&-\neg F_{2} \Rightarrow F_{2}, F_{2} \&-\neg F_{3} \Rightarrow F_{3}\right\} \not$ $F_{1} \&-\neg F_{3} \Rightarrow F_{3}$.

Interestingly, rule calculus is monotonic although the extension semantics of default logic is dealing with nonmonotonicity.

Proposition 16 (Monotonicity). Let $R$ be a rule and $\Delta$ and $\Delta^{\prime}$ are two rule bases such that $\Delta \subseteq \Delta^{\prime}$. If $\Delta \vdash R$, then $\Delta^{\prime} \vdash R$.

Theorem 2 (Complexity). Checking whether a rule $R$ has at least one HT-model is NP complete. 
Proof. Hardness is obvious since a fact is satisfiable iff it has at least one HT-model. For membership, we first prove a lemma by induction. Given two HT-interpretations $\left\langle T_{1}, T_{2}\right\rangle$ and $\left\langle T_{1}^{\prime}, T_{2}^{\prime}\right\rangle$ and a set of facts $\Gamma$, if for all $F \in \Gamma, T_{1} \models F$ iff $T_{1}^{\prime} \models F$, and so do $T_{2}$ and $T_{2}^{\prime}$, then for all rules $R$ composed from $\Gamma$ and rule connectives, $\left\langle T_{1}, T_{2}\right\rangle \models R$ iff $\left\langle T_{1}^{\prime}, T_{2}^{\prime}\right\rangle \models R$.

Suppose that $R$ is composed from the set of facts $\Gamma=\left\{F_{1}, \ldots, F_{n}\right\}$ and $\left\langle T_{1}, T_{2}\right\rangle \models$ $R$. Without loss of generality, suppose that $T_{1} \models F_{i},(1 \leq i \leq m)$ and $T_{1} \not \models F_{i},(m<$ $i \leq n)$. Therefore, there exists a propositional assignment $\pi_{i},(m<i \leq n)$ such that $\pi_{i} \models \bigwedge_{1 \leq j \leq m} F_{j} \wedge \neg F_{i}$. Let $T_{1}^{\prime}$ be the theory such that its models are $\pi_{i},(m<i \leq n)$. It is easy to see that $T_{1}$ and $T_{1}^{\prime}$ agree the same on $\Gamma$. We can construct $T_{2}^{\prime}$ in the same way. We have that $T_{1}^{\prime} \subseteq T_{2}^{\prime}$. Therefore $\left\langle T_{1}^{\prime}, T_{2}^{\prime}\right\rangle \models R$. This shows that if a rule $R$ has a model, then it has a model which can be represented polynomially. It follows that checking whether a rule $R$ has at least one HT-model is in NP.

It is well known that most of the decision problems in default logics lie on the second level of polynomial hierarchy [6], even restricted to some special subclasses [5]. Surprisingly, although rule calculus seems more complicated than others such as skeptical and credulous reasoning, its complexity is lower than them according to Theorem 2. This draws an opposite conclusion. That is, the problem of rule calculus is, indeed, simpler than other reasoning tasks of default logic. However, this does not mean that the former is weaker than the latter since they are dealing with different reasoning tasks of default rules.

\section{Applications}

Since the notion of rule deduction is an extension of deduction in propositional calculus, many propositional logic based deductive reasoning tasks can be lifted to corresponding cases in rule calculus. In this section, we briefly discuss three applications, which seem to be hard to deal with in default logic on their own. However, by using rule calculus, these problems can be easily solved. Due to a space limit, we only outline the basic ideas here.

\section{Irrelevance in default logic}

As pointed by Lang et al. [13], irrelevance is an important notion in propositional logic. According to Lang et al.'s definition, a propositional formula $F$ is irrelevant to a set $V$ of atoms iff there exists another formula $G$ such that $F$ is equivalent to $G$ and $\operatorname{Atom}(G) \cap$ $V=\emptyset$, where $\operatorname{Atom}(G)$ is the set of atoms appeared in $G$. The notion of irrelevance in rule calculus can be defined in a similar way. That is, a rule $R_{1}$ is irrelevant to a set $V$ of atoms iff there exists another rule $R_{2}$ such that $\models R_{1} \Leftrightarrow R_{2}$ and $\operatorname{Atom}\left(R_{2}\right) \cap V=\emptyset$, where $\operatorname{Atom}\left(R_{2}\right)$ is the set of atoms occurred in $R_{2}$.

Having defined the notion of irrelevance in rule calculus, we can define other related notions such as forgetting in a similar way as shown in [13].

\section{Generality among default rules}

The concept of generality is a foundational basis of inductive logic programming [14, 15] - a subfield of machine learning and has been successfully applied to some real 
domains. Inductive logic programming started from propositional logic [14] but then has focused on Horn clauses (namely logic programs) [15].

In propositional logic, the generality relationships between two formulas can be defined in a simple way as shown in [14]. That is, a formula $F_{1}$ is said to be more general than a formula $F_{2}$ iff $F_{1} \models F_{2}$ and $F_{2} \not \models F_{1}$. We can lift this notion to the case between two rules. A rule $R_{1}$ is said to be more general than a formula $R_{2}$ iff $R_{1} \models R_{2}$ and $R_{2} \not \models R_{1}$. Moreover, this notion can be easily extended to the cases with a background rule base. A rule $R_{1}$ is said to be more general than a rule $R_{2}$ relative to a rule base $\Delta$ iff $\Delta \cup\left\{R_{1}\right\} \models R_{2}$ and $\Delta \cup\left\{R_{2}\right\} \not \models R_{1}$. It is obvious that this definition is a generalization of the definition of generality in propositional calculus since all propositional formulas are also rules.

Inoue and Sakama [16] introduced several kinds of generality relationship between default theories. Although their definitions are based on disjunctive default logic, these can be easily extended to general default logic. We write $\operatorname{Ext}(R)$ to denote the set of extensions of a rule $R$. Let $R_{1}$ and $R_{2}$ be two rules, according to Inoue and Sakama's definitions, $R_{1}$ is said to be more $\sharp$-general than $R_{2}$ iff for all $T_{1} \in \operatorname{Ext}\left(R_{1}\right)$, there exists $T_{2} \in \operatorname{Ext}\left(R_{2}\right)$ such that $T_{2} \subseteq T_{1} ; R_{1}$ is said to be more b-general than $R_{2}$ iff for all $T_{2} \in \operatorname{Ext}\left(R_{2}\right)$, there exists $T_{1} \in \operatorname{Ext}\left(R_{1}\right)$ such that $T_{2} \subseteq T_{1} ; R_{1}$ is said to be strongly more $\sharp$-general than $R_{2}$ iff for all rules $R_{3} R_{1} \& R_{3}$ is more $\sharp$-general than $R_{2} \& R_{3} ; R_{1}$ is said to be strongly more b-general than $R_{2}$ iff for all rules $R_{3} R_{1} \& R_{3}$ is more b-general than $R_{2} \& R_{3}$. However, the cases relative to a background default theory were not considered in their approach.

Our definition of generality does not coincide with any of these notions. For instance, let $p_{1}$ and $p_{2}$ be two atoms. We have that $T$ is both $\sharp$-general and b-general than $-p_{1}$ but the former is not more general than the latter in our definition. Meanwhile, $p_{1} \wedge p_{2}$ is more general than $p_{1}$ in our definition but the former is neither strongly more $\sharp$-general nor strongly b-general than the latter. One major difference between these two approaches is that Inoue and Sakama's notions are defined based on the sets of extensions of default theories. However, two rules sharing the same set of extensions may play completely different roles in rule calculus.

\section{Revising default rule bases}

Belief revision has been an important topic in solving information conflict in reasoning about agents. In most existing approaches and systems, an agent's knowledge base is usually represented by a set of classical propositional formulas, then various revision methods have been developed by researchers to solve the inconsistency by revising a knowledge base by a new piece of information.

Under the framework of rule calculus, this work can be generalized to nonmonotonic knowledge base revision. That is, in our setting, each agent's knowledge is represented as a rule base, and the problem is how to revise this rule base by giving a new default rule.

We may specify a formulation of rule base revision by generalizing approaches for propositional belief revision, for instance, the WIDTIO approach [17]. Given a rule base $\Delta$ and a rule $R$, we say that $\Delta^{\prime}$ is a maximal subset of $\Delta$ consistent with $R$ iff a) $\Delta^{\prime} \subseteq \Delta$, b) $\Delta^{\prime} \cup\{R\}$ is not a rule contradiction, and c) there does not exist $\Delta^{\prime \prime}$ 
satisfying the above two conditions and $\Delta^{\prime} \subset \Delta^{\prime \prime} \subseteq \Delta$. The rule base revision operator $\circ$ is defined as $\Delta \circ R=\bigcap \Delta^{\prime} \cup\{R\}$. This revision operator also satisfies the well-known AGM postulates.

\section{Conclusion}

In this paper, we extend the logic of here-and-there as a general semantics for default rules. Meanwhile, we propose a corresponding axiom system for rule calculus and prove the soundness and completeness theorem (see Theorem 1). We also discuss other properties in rule calculus, including complexity issues (see Theorem 2).

The notion of strong equivalence in default logic can be directly captured in rule calculus (See Corollary 1). Corollary 1 also indicates the intuition behind rule deduction, that is, a rule $R$ is a consequence of a rule base $\Delta$ means that $R$ provides no more information by giving $\Delta$. In other words, $R$ can be eliminated by giving $\Delta$. On the other hand, given the fact that answer set programming is a special case of default logic, our approach also shows that the logic of here-and-there and its axiomatic counterpart G3 can capture the consequence relationships among answer set programs. In fact, restricted to answer set programs (i.e., facts are atoms instead of arbitrary propositional formulas), rule calculus coincides with the notion of SE-consequence [18, 19].

Rule calculus is an extension of propositional calculus and also an extension of the intermediate logic G3 [7] in the sense that the connectives in G3 are represented as rule connectives. It can also be considered as an extended logic of formalizing normality [20] since the sentence " $A$ normally implies $B$ " can be represented as $A \&-\neg B \Rightarrow B$ as suggested by Reiter [4].

Rule calculus is different from conditional logic [21] although both of them introduce new connectives into propositional calculus. There are two syntactic differences. First, conditional logic only introduces a conditional connective $>$. Second, whereas conditional logic allows arbitrary compositions of atoms and connectives including $>$, in most cases, it uses $>$ as a lower level connective, whilst in rule calculus, classical connectives are at the lower level. Certainly, the axiom systems and semantics of these two logics are basically dissimilar. For instance, the conditional connective $>$ is intuitively stronger than $\rightarrow$ in conditional logic, whilst the rule implication $\Rightarrow$ is, to some extent, weaker than $\rightarrow$ in rule calculus.

Another related work is so-called proof theory of default logic [22,23], which aims to define a proof-theoretical system for determining whether a propositional formula is in all (or some) extensions of a default theory. It differs from rule calculus in several aspects. Firstly, proof theory of default logic is operating on the level of extension semantics, whilst rule calculus is focused on the here-and-there semantics. Secondly, The consequence concerned in rule calculus is, in general, default rules instead of propositional formulas. Finally, even restricted to the cases of facts, as we mentioned earlier (See Proposition 2), these two systems do not coincide with each other. 


\section{Acknowledgement}

This work is supported by Australian Research Council (ARC) Discovery Projects grant DP0666540. The authors thank the anonymous reviewers for their valuable comments.

\section{References}

1. Gelfond, M., Lifschitz, V., Przymusinska, H., Truszczyński, M.: Disjunctive defaults. In: Proceedings of the KR'91. (1991) 230-237

2. Turner, H.: Strong equivalence for logic programs and default theories (made easy). In: Proceedings of the LPNMR '01. (2001) 81-92

3. Zhou, Y., Lin, F., Zhang, Y.: General default logic. In: Proceedings of the LPNMR'07. (2007) 241-253

4. Reiter, R.: A logic for default reasoning. Artificial Intelligence 13 (1980) 81-132

5. Ben-Eliyahu-Zohary, R.: Yet some more complexity results for default logic. Artificial Intelligence 139(1) (2002) 1-20

6. Gottlob, G.: Complexity results for nonmonotonic logics. Journal of Logic and Computation 2(3) (1992) 397-425

7. Jongh, D.H.J.D., Hendriks, L.: Characterization of strongly equivalent logic programs in intermediate logics. Theory and Practice of Logic Programming 3(3) (2003) 259-270

8. Cabalar, P., Ferraris, P.: Propositional theories are strongly equivalent to logic programs. Theory and Practice of Logic Programming 7(6) (2007) 745-759

9. Ferraris, P.: Answer sets for propositional theories. In: Proceedings of the LPNMR'2005. (2005) 119-131

10. Pearce, D.: A new logical characterisation of stable models and answer sets. In: Proceedings of NMELP '96. (1997) 57-70

11. Lifschitz, V., Pearce, D., Valverde, A.: Strongly equivalent logic programs. ACM Transactions on Computational Logic 2(4) (2001) 526-541

12. van Dalen, D.: Intuitionistic logic. In Gabbay, D., Guenthner, F., eds.: Handbook of Philosophical Logic: Volume III: Alternatives to Classical Logic. (1986) 225-339

13. Lang, J., Liberatore, P., Marquis, P.: Propositional independence: formula-variable independence and forgetting. Journal of Artificial Intelligence Research (JAIR) 18 (2003) 391-443

14. Plotkin, G.: A note on inductive generalization. In Meltzer, B., Michie, D., eds.: Machine Intelligence. (1970) 153-163

15. Muggleton, S.: Inductive logic programming. In: The MIT Encyclopedia of the Cognitive Sciences (MITECS). MIT Press (1999)

16. Inoue, K., Sakama, C.: Generality and equivalence relations in default logic. In: Proceedings of the AAAI'07. (2007) 434-439

17. Winslett, M.: Updating logical databases. Cambridge University Press (1990)

18. Eiter, T., Fink, M., Tompits, H., Woltran, S.: Simplifying logic programs under uniform and strong equivalence. In: LPNMR. (2004) 87-99

19. Wong, K.: Sound and complete inference rules for se-consequence. Journal of Artificial Intelligence Research 31 (2008) 205-216

20. Geffner, H., Pearl, J.: Conditional entailment: bridging two approaches to default reasoning. Artificial Intelligence 53(2-3) (1992) 209-244

21. Nute, D.: Conditional logic. In Gabbay, D., Guenthner, F., eds.: Handbook of Philosophical Logic: Volume II: Extensions of Classical Logic. (1984) 387-439

22. Bonatti, P.A., Olivetti, N.: A sequent calculus for skeptical default logic. In: Proceedings of the TABLEAUX '97. (1997) 107-121

23. Lakemeyer, G., Levesque, H.J.: Towards an axiom system for default logic. In: Proceedings of the AAAI'06. (2006) 